\section{Colour your life in the lab}

Spring has sprung with a range of new colourful tools for protein expression, chromosome 'painting' and telomere-labelling, in addition to faster strategies for CDNA synthesis and gel-loading.

The latest buzz in transfection systems is Invitrogen's Drosophila Expression System (DESTM), which provides a less expensive option to expression

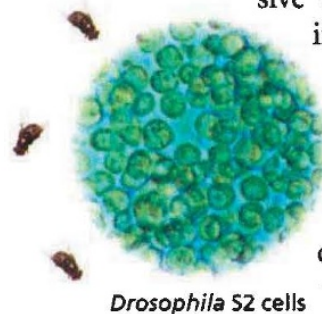

in mammalian cells and a more efficient alternative to using baculovirus. A range of vectors enables constitutive, inducible or secreted expression of target-gene products in Drosophila S2 cells. Each vector also provides a C-terminal fusion tag encoding the $\mathrm{V} 5$ epitope for simple detection using the Anti-V5 Antibody ${ }^{\mathrm{TM}}$ and a polyhistidine sequence for rapid purification with ProBond ${ }^{\mathrm{TM}}$ resin.

Enjoy protein expression in technicolour with CLONTECH's Enhanced Yellow Fluorescent protein (EYFP). This yellow-green variant of the Aequorea victoria green fluorescent protein (GFP) contains a four-aminoacid substitution that shifts the fluorescence emission from green $(509 \mathrm{~nm})$ to yellowish green $(527 \mathrm{~nm})$, allowing flow-cytometric sorting of cells expressing the different GFP colour variants. Optimized for high expression in mammalian and plant cells, this new 'living colour' product provides more flexibility when selecting a variant for simultaneous analysis of different promoter elements, intracellular localization of various proteins and interactions between protein fusions. If blue and green are your favourite colours of the rainbow, Quantum Biotechnologies has created even more brilliant Blue and Green AutoFluorescent Proteins ${ }^{\mathrm{T} M}$ to brighten your day under the microscope.

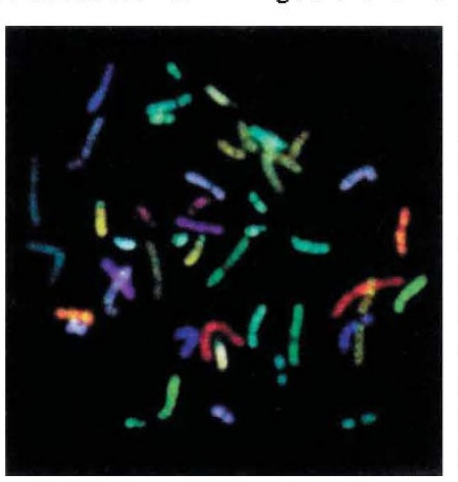

Vysis' Quips mFISH imaging software enables 'painting' of all 24 human chromosomes in a single cell

For those 'tough-totransfect' cells, Boehringer Mannheim places a new arrow in its quiver, with FuGene ${ }^{\mathrm{TM}}$ Transfection Reagent. This cocktail of nonliposomal lipids penetrates a range of cell types including Drosophila S2 cells, primary cultures, HeLa, 3T3 and COS-1 cells. The reagent apparently delivers with high-efficiency and low cytotoxicity and is compatible with the presence of serum in the media.

For those feeling fickle, Stratagene now offers maximal flexibility in shuttling between eukaryotic and prokaryotic expression systems with the release of the $\mathrm{pDUAL} \mathrm{L}^{\mathrm{TM}}$ vector. The vector contains the human $\mathrm{CMV}$ immediate early gene promoter for constitutive expression in mammalian cells. A hybrid T7/lacO promoter regulates transcription in bacteria and the presence of the lacI repressor gene allows inducible expression with IPTG. A distinguishing feature of pDual is the presence of the bacterial ShineDalgarno and mammalian Kozak consensus sequences to ensure efficient translation of the cloned gene in either system. Depending on the reverse primer used in the PCR cloning into pDual, researchers can choose to have the target gene product expressed in its native form or, alternatively, fuse it to a small calmodulin binding peptide tag for detection and purification using Stratagene's Affinity ${ }^{\mathrm{TM}}$ CBP fusion detection kit and Calmodulin Affinity Resin, respectively.

A new vision in chromosome-imaging has arrived with the Quips ${ }^{T M}$ mFISH imaging software from Vysis. Chromosomes are stained with a set of targetspecific probes, each labelled with one of seven different fluorophores. The Quips software then captures the seven different colour planes and implements clever computational colour-blending and combinatorial labelling techniques to transform the 24 human chromosomes into a palette of colour. The software system, which supports both automated and manual microscopes, enables the detection of several genetic aberrations within a single cell. For 'painting' the tips of chromosomes, Cytocell offers the Chromoprobe Multiprobe ${ }^{\circledR}$ T system, a set of 41 telomere-specific FISH probes, for simulta- neous analysis of the subtelomeric regions of every chromosome.

If you'd rather enjoy spring outdoors, CPG takes you from tissues and cells to an immobilized cDNA library in two hours or less with the SOLIDscript $^{\mathrm{T} \mathrm{M}}$ Solid Phase

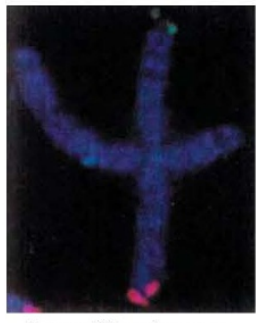

Cytocell's telomerespecific FISH probes cDNA synthesis kit.

Following homogenization of cells or tissues, the mRNA is captured with oligo (dT)linked magnetic porous glass (MPG ${ }^{\$}$ ) particles. The mRNA is then reverse-transcribed, using the immobilized oligo dT to prime the reaction, thus generating an immobilized cDNA library. The entire process takes place in a single tube, thereby eliminating potential loss of the template. The CDNA library can be used directly in PCR, RACE, subtractive hybridisation and CDNA cloning.

PCR has gone platinum. Life Technologies' PLATINIUM $^{\text {TM }}$ Taq Antibody mixture has been developed to target specific epitopes of Taq polymerase to enhance performance and completely inhibit enzyme activity until the first denaturation temperature is reached, thereby eliminating any chance of pre-PCR mispriming. In conjunction with PLATINIUM $^{T M}$ Taq polymerase, the system apparently offers higher specificity, yield and fidelity of PCR amplification.

For all those sequencers weary of leaking wells and broken gel walls, The Gel Company now offers 'Rapid Load' membrane combs to make gel-loading almost a joy. Samples are aliquoted into the wells of a tray and then a porous comb is 'dipped' into the a tray to absorb the samples simultaneously. (Alternatively, the samples can be loaded on the teeth of the comb directly using single or multichannel pipette tips.) The comb is then inserted between the glass plates and the samples electrophoresed into the gel. The load volume per tooth has a recommended limit of $1.0 \mu \mathrm{l}$, but combs capable of larger sample volumes are currently under development. Finally, Stratagene gets those dyes right out of your gel buffers with the Eliminator ${ }^{\mathrm{TM}}$. This environmentally-friendly dye-removal system eliminates more than $99 \%$ of ethidium bromide from solutions, ensuring that the 'colour' stays in your lab, rather than flowing down the sink.

Notes compiled by Carina Dennis 